\title{
REVISTAS CIENTÍFICAS: ESTÁNDARES EN REVISTAS CIENTÍFICAS EN LÍNEA
}

\section{Authier, Carlos Norberto}

\section{Resumen:}

Aborda los estándares de revistan en línea. Las bases de datos y sus tipos. Hace hincapié en Latindex (Sistema Regional de Información en Línea para Revistas Científicas de América Latina, el Caribe, España y Portugal) y dos de sus productos: Directorio y Catálogo. Hace una descripción del Núcleo Básico de Revistas Científicas Argentinas.

Palabras clave: Indización, Bases de datos, Latindex, Núcleo Básico de Revistas Científicas Argentinas

\section{INTRODUCCION}

Las revistas científicas en el siglo XXI deben cumplir con ciertos estándares para lograr la visibilidad de sus contenidos. En la actualidad las revistas científicas, en su gran mayoría, están en línea para que sus contenidos puedan ser visualizados y descargados en cualquier parte del mundo. Aparte de estar en línea deben tener ciertas características pedidas por las bases de datos de indización para poder ingresar a ellas.

\section{BASES DE DATOS DE INDIZACIÓN}

Facilitan la búsqueda de la revistas, de las investigaciones científicas y la diseminación de la producción científica.

\section{TIPOS}

Cada base de datos de indización tiene su propio criterio para incluir revistas y también ofrece información restringida de acuerdo al contenido de la información que procesa y al tipo de usuarios al que está orientada. Actualmente la información de los requisitos de cada base de datos está explicitada en su página web y puede cambiar rápidamente por lo que se recomienda leer y estudiar los requisitos de ingreso al momento de presentarse a una base de datos para ser incluida en la misma. Es de destacar que las bases cambian a lo largo del tiempo, algunas crecen, otras pierden financiación y dejan de funcionar, otras hacen acuerdos con otras bases y finalmente surgen nuevas con mayor velocidad de cosecha de datos (utilizan procesos automatizados para levantar la información de las revistas incluidas). 


\section{DIRECTORIO DE LATINDEX}

Puede encontrarse en este link: https://www.latindex.org/latindex/inicio

El ingreso a Latindex Directorio es muy sencillo, se debe ingresar un formulario por cada soporte que tiene la revista (comúnmente impreso y electrónico). En caso de tener un formulario para la revista impresa se debe ingresar uno nuevo aquí para la revista electrónica:

https://www.latindex.org/latindex/formReg

Al enviarlo se despliega un formulario que debe ser completado lo más detallado posible.

\section{CATÁLOGO DE LATINDEX}

Puede encontrarse en este link: https://www.latindex.org/latindex/inicio

Con respecto a Latindex Catálogo se requiere previamente haber ingresado la revista a Latindex Directorio.

Luego se recomienda leer el nuevo procedimiento de calificación:

https://www.latindex.org/latindex/meto2

En caso de considerar que la revista cumple más de 30 características de las 38 posibles, se puede solicitar un formulario de autoevaluación a la Coordinación de Latindex Argentina:

https://www.latindex.org/latindex/coordinadores

Este formulario es revisado y luego la revista es dada de alta en Latindex Catálogo 2.0 .

En caso de cumplir una nueva característica faltante vuelven a comunicarse para que la página de Latindex sea modificada por la Coordinación de Latindex Argentina.

\section{NÚCLEO BÁSICO DE REVISTAS CIENTÍFICAS ARGENTINAS}

En este link se actualiza la información: http://www.caicyt-conicet.gov.ar/sitio/comunicacion-cientifica/nucleo-basico/

A diferencia de Latindex Catálogo: "El Núcleo Básico de Revistas Científicas Argentinas determina un conjunto de publicaciones científicas y tecnológicas argentinas de excelencia, en los distintos campos del conocimiento. Dichas publicaciones científicas son sometidas a una evaluación exhaustiva con criterios únicos definidos de calidad y trascendencia, acorde con criterios internacionales, establecido por la Resolución 1640/05 de CONICET."

Además de una evaluación técnica como la de Latindex Catálogo las revistas son evaluadas por los miembros del Comité Científico Asesor por especialistas en las distintas disciplinas. 


\section{OTRAS BASES}

Existen varios cientos de bases de datos que indexan las revistas científicas. Las mismas pueden ser consultadas en el CAICYT (Centro Argentino de Información Científica y Tecnológica) con el Área Comunicación Institucional o el Área Comunicación Científica:

http://www.caicyt-conicet.gov.ar/sitio/

\section{REFERENCIAS BIBLIOGRÁFICAS}

Abejón Peña, Teresa; Alonso Gamboa, José Octavio; Córdoba González, Saray y Polanco Cortés, Jorge. (2019). Latindex. México: Latindex Sistema Regional de Información en Línea para Revistas Científicas de América Latina, el Caribe, España y Portugal. Recuperado de https://www.latindex.org/latindex/meto2. Consultado el 19/09/2019.

Licencia: Attribution-NonCommercial-ShareAlike 4.0 International (CC BY-NC-SA 4.0) 